\title{
Prognostic Significance of a Micropapillary Pattern in Pure Mucinous Carcinoma of the Breast: Comparative Analysis with Micropapillary Carcinoma
}

\author{
Hyun-Jung Kim • Kyeongmee Park \\ Jung Yeon Kim · Guhyun Kang \\ Geumhee Gwak ${ }^{1}$. Inseok Park ${ }^{1}$ \\ Departments of Pathology and ${ }^{1}$ Surgery, Inje \\ University Sanggye Paik Hospital, Seoul, Korea
}

\author{
Received: January 15, 2017 \\ Revised: February 27, 2017 \\ Accepted: March 14, 2017 \\ Corresponding Author \\ Kyeongmee Park, MD, PhD \\ Department of Pathology, Inje University Sanggye \\ Paik Hospital, 1342 Dongil-ro, Nowon-gu, \\ Seoul 01757, Korea \\ Tel: +82-2-950-1263 \\ Fax: +82-2-951-6964 \\ E-mail: kpark@paik.ac.kr
}

\begin{abstract}
Background: Mucinous carcinoma of the breast is an indolent tumors with a favorable prognosis; however, micropapillary features tend to lead to aggressive behavior. Thus, mucinous carcinoma and micropapillary carcinoma exhibit contrasting biologic behaviors. Here, we review invasive mucinous carcinoma with a focus on micropapillary features and correlations with clinicopathological factors. Methods: A total of 64 patients with invasive breast cancer with mucinous or micropapillary features were enrolled in the study. Of 36 pure mucinous carcinomas, 17 (47.2\%) had micropapillary features and were termed mucinous carcinoma with micropapillary features (MUMPC), and 19 (52.8\%) had no micropapillary features and were termed mucinous carcinoma without micropapillary features. MUMPC were compared with 15 invasive micropapillary carcinomas (IMPC) and 13 invasive ductal and micropapillary carcinomas (IDMPC). Results: The clinicopathological factors of pure mucinous carcinoma and MUMPC were not significantly different. In contrast to IMPC and IDMPC, MUMPC had a low nuclear grade, lower mitotic rate, higher expression of hormone receptors, negative human epidermal growth factor receptor 2 (HER2) status, lower Ki-67 proliferating index, and less frequent lymph node metastasis $(\mathrm{p}<.05)$. According to univariate analyses, progesterone receptor, HER2, T-stage, and lymph node metastasis were significant risk factors for overall survival; however, only T-stage remained significant in a multivariate analysis $(p<.05)$. Conclusions: In contrast to IMPC and IDMPC, the micropapillary pattern in mucinous carcinoma does not contribute to aggressive behavior. However, further analysis of a larger series of patients is required to clarify the prognostic significance of micropapillary patterns in mucinous carcinoma of the breast.
\end{abstract}

Key Words: Breast; Micropapillary; Mucinous; Prognosis
Mucinous carcinoma of the breast, believed to be an indolent tumor, affects older females. Pure mucinous carcinoma with a $>90 \%$ mucinous component has a better prognosis than that of mixed mucinous carcinoma. This carcinoma tends to follow an indolent course with infrequent lymphatic or hematogenic dissemination and to have a favorable prognosis. ${ }^{1-4}$ Invasive micropapillary carcinoma (IMPC) was first reported by Siriaunkgul and Tavassoli ${ }^{5}$ in 1993 as a rare subtype of breast carcinoma. IMPC is characterized by tumor cells arranged in tubules with a small or obliterated lumen, which extensively penetrate the lymphatic or vascular space, leading to a high frequency of lymph node metastasis. $^{6-14}$ In a previous study, axillary lymph node metastases were found in all of 27 patients at the initial diagnosis. Twelve of these patients were followed up, and six died at a mean of 22 months after the initial treatment. ${ }^{12}$ It is important for pathologists to identify micropapillary formations, as they indicate the potential for aggressive tumor behavior and influence the choice of therapy. Thus, the micropapillary and pure mucinous subtypes of invasive carcinoma are opposites in terms of their biological behavior. ${ }^{15}$ These two patterns sometimes coexist in the same tumor. Tumor cells in mucinous carcinoma can exhibit different patterns, such as cords, trabeculae, cribriform structures, and solid lobules. Previous studies have suggested that the micropapillary pattern in cases of mucinous carcinoma is associated with aggressive tumor behavior. ${ }^{16-18}$ The pathogenetic association of this mucinous micropapillary carcinoma (MUMPC) with pure mucinous carcinoma is unclear.

The aim of this study was to characterize the clinicopathological parameters of MUMPC and to compare them with those of pure mucinous carcinoma, IMPC, and invasive ductal and micropapillary carcinoma (IDMPC). Moreover, the prognostic significance of micropapillary features in breast carcinoma was 
determined.

\section{MATERIALS AND METHODS}

We analyzed 64 patients diagnosed with MUMPC, IMPC, IDMPC, or mucinous carcinoma without micropapillary features (MUC). These 64 cases were diagnosed and treated for breast carcinoma at Inje University Sanggye Paik Hospital between 1997 and 2012. Only cases with tumors containing a $\geq 90 \%$ mucinous component were defined as mucinous carcinoma. Morphologically, micropapillae were defined as clusters of tumor cells separated from surrounding stroma by clear spaces and that exhibited reverse polarity, also known as an "inside-out" growth pattern, whereby the apical pole of the cells faces the stroma rather than the luminal surface. ${ }^{19}$ MUMPC was defined as a tumor in which micropapillary features constituted $>50 \%$ of the tumor epithelial components (Fig. 1). Patient age, tumor stage by TNM classification, ${ }^{20}$ lymph node metastasis, nuclear grade, mitotic rate, estrogen receptor (ER) status, progesterone receptor (PR) status, human epidermal growth factor receptor 2 (HER2) status, Ki-67 proliferating index, molecular subtype, and survival were recorded. The following antibodies were used for immunohistochemistry (IHC): ER (SP1, Dako, Carpinteria, CA, USA), PR (SP2, Dako), HER2 (HercepTest, Dako), Ki-67 (MIB-1, Dako), cytokeratin (CK) 5/6 (D5/16, Dako), and epidermal growth factor receptor (EGFR; E30, Dako). Cases were classified as ER-, PR-, and HER2-positive or negative, and the latest American Society of Clinical Oncology guidelines for the detection and scoring of ER, PR, and HER2 were followed. ${ }^{21,22}$ Cases with a HER2 IHC grade of $2+$ subsequently underwent evaluation of HER2 amplification by fluorescence in situ hybridization (PathVysion, Abbott, Des Plaines, IL, USA) and were designated as positive or negative. Ki-67 expression was classified as low $(<14 \%)$ or high $(\geq 14 \%){ }^{23}$ Based on the IHC results, molecular subtypes were

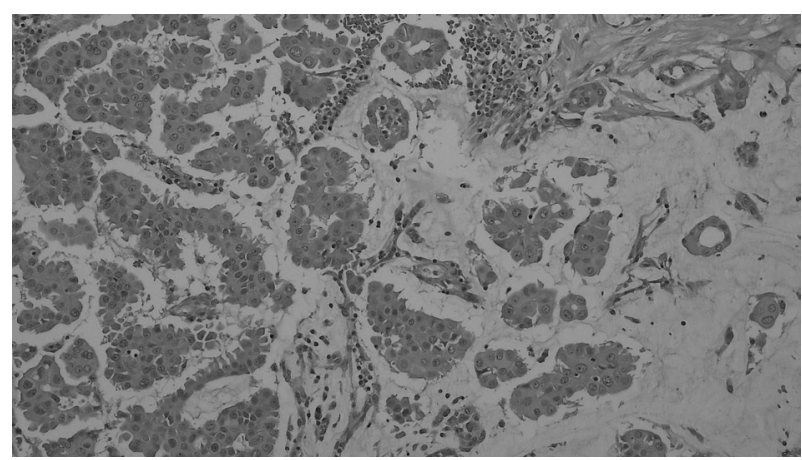

Fig. 1. Micropapillary features were found in more than $50 \%$ of the mucinous carcinoma cases. defined as follow: luminal $\mathrm{A}\left(\mathrm{ER}^{+}\right.$and/or $\mathrm{PR}^{+}, \mathrm{HER} 2^{-}, \mathrm{Ki}-67$ low), luminal $\mathrm{B}$ (ER ${ }^{+}$and/or $\mathrm{PR}^{+}, \mathrm{HER} 2^{+} / \mathrm{ER}^{+}$and/or $\mathrm{PR}^{+}$, HER2 ${ }^{-}$, Ki-67 high), HER2-positive (ER ${ }^{-}, \mathrm{PR}^{-}, \mathrm{HER} 2^{+}$), and basal-like $\left(\mathrm{ER}^{-}, \mathrm{PR}^{-}, \mathrm{HER} 2^{-}, \mathrm{EGFR}^{+}\right.$and/or $\left.\mathrm{CK} 5 / 6^{+}\right){ }^{23}$ Patients underwent either mastectomy or breast conserving surgery with lymph node dissection. Adjuvant chemotherapy or hormonal therapy was routinely considered. All patients underwent mammography, physical examination, chest X-ray, and breast ultrasonography during follow-up of 6 to 96 months. This study was approved by the Institutional Review Board of our hospital (SGPAIK 2016-04-002).

The following clinical and pathological factors were assessed: age at diagnosis, nuclear grade (a one-level increase on a 1-3 scale), mitotic rate (a one-level increase on a 1-3 scale), ER expression (positive vs negative), PR expression (positive vs negative), HER2 amplification (positive vs negative) and the Ki-67 proliferation index (high vs low). Molecular tumor subtypes were categorized as luminal A, luminal B, basal-like, or HER2 positive. The clinicopathological features of MUC, MUMPC, IMPC, and IDMPC cases were compared. Pearson's chi-square test, Student's t test, and ANOVA were used to evaluate discrete and quantitative variables. Among the clinicopathological variables, the potential risk factors for overall survival (OS) were examined using univariate and multivariate logistic regression analyses. Statistical analyses were performing using IBM SPSS Statistics ver. 20 software (IBM Corp., Armonk, NY, USA). Statistical significance was determined using the likelihood ratio test and accepted at values of $\mathrm{p}<.05$.

\section{RESULTS}

The 64 cases comprised 19 (29.7\%) with MUC, 17 (26.6\%) with MUMPC, 15 (23.4\%) with IMPC, and 13 (20.3\%) with IDMPC. All cases were female and aged 29-79 years (mean, $51.8 \pm 12.8$ years).

Both MUC and MUMPC showed low nuclear grades and low mitotic rates. In both groups, all tumor cells were positive for ER, irrespective of the presence of micropapillary features. All but one of the MUC cases revealed strong PR expression but no HER2 amplification. All but one of the MUMPC cases were positive for PR and negative for HER2 amplification. A high Ki-67 proliferation index was noted in eight cases of MUC (42.1\%) and 10 cases of MUMPC (58.8\%). Among the MUC and MUMPC cases, 11 (57.9\%) and seven cases (41.2\%) were luminal A, respectively, and eight (42.1\%) and 10 cases (58.8\%) were luminal B. Neither the HER2 nor basal-like subtype was detected in MUC or MUMPC. Among the MUC cases, 11 cases (57.5\%) 
were T1, seven cases (36.8\%) were T2, and a case (5.3\%) was T3. Among the MUMPC, six cases $(35.3 \%)$ were T1, seven cases (41.2\%) were T2, and four cases (23.5\%) were T3. Metastasis to the axillary lymph node was detected in two cases of MUC (10.5\%) and four cases of MUMPC (23.5\%). The surrounding breast parenchyma showed changes suggestive of ductal carcinoma in situ in 13 cases $(36.1 \%)$, comprising eight cases of MUC and five of MUMPC (Table 1).

Compared with IMPC and IDMPC, nuclear grade and mitotic rate were lower in MUMPC $(\mathrm{p}<.01)$. MUMPC showed lower frequency of ER and PR negativity $(\mathrm{p}=.04)$ and HER2 positivity $(\mathrm{p}=.01)$. The Ki-67 proliferation index of the MUMPC cases was lower than that of the IMPC and IDMPC cases $(\mathrm{p}<.01)$. Upon comparison of MUC and MUMPC, IMPC and IDMPC revealed less frequent luminal $A$ and more frequent HER2 and basal-like subtypes $(\mathrm{p}<.01)$. Among the MUMPC cases, six cases $(35.3 \%)$ were $\mathrm{T} 1$, seven cases $(41.2 \%)$ were $\mathrm{T} 2$, and four cases $(23.5 \%)$ were T3. Among the IMPC and IDMPC cases, six cases $(40.0 \%)$ and four cases $(30.8 \%)$ were $\mathrm{T} 1$, six cases $(40.0 \%)$ and seven cases $(53.8 \%)$ were T2, and three cases $(20.0 \%)$ and two cases (15.4\%) were T3, respectively. Four of the MUMPC cases $(23.5 \%)$ exhibited lymph node involvement, while metastasis to the axillary lymph node was detected in $12(80.0 \%)$ and nine $(69.2 \%)$ cases of IMPC and IDMPC, respectively $(\mathrm{p}<.01)$ (Table 1). The surrounding breast parenchyma showed changes suggested of ductal carcinoma in situ in 19 cases (67.9\%), comprising eight cases of IMPC and 11 of IDMPC.

Mean follow-up duration was $83 \pm 51$ months. Overall, PR and HER2 statuses, T-stage, and lymph node metastasis were correlated with death (Table 2). Univariate analysis using a logistic regression model revealed that OS was significantly associated with PR and HER2 statuses, T-stage, and lymph node metastasis (Table 3). However, on multivariate analysis, only T-stage was significantly associated with OS (Table 3).

\section{DISCUSSION}

Pure mucinous carcinoma of the breast has a good prognosis with a low rate of regional lymph node metastasis and excellent survival, in contrast to invasive ductal carcinoma of no special

Table 1. Comparisons of clinicopathological parameters among MUC, MUMPC, IMPC, and IDMPC

\begin{tabular}{|c|c|c|c|c|c|c|}
\hline Parameter & & MUC $(n=19)$ & MUMPC $(n=17)$ & IMPC $(n=15)$ & IDMPC $(n=13)$ & $p$-value ${ }^{a}$ \\
\hline Age, mean (yr) & & $52.7 \pm 14.0$ & $53.9 \pm 11.8$ & $45.9 \pm 11.7$ & $54.3 \pm 12.6$ & $.22^{b}$ \\
\hline \multirow[t]{3}{*}{$N G$} & Low & $6(31.6)$ & $1(5.9)$ & 0 & 0 & $<.01$ \\
\hline & Intermediate & $11(57.9)$ & $14(82.4)$ & $4(26.7)$ & $4(30.8)$ & \\
\hline & High & $2(10.5)$ & $2(11.8)$ & 11 (73.3) & $9(69.2)$ & \\
\hline \multirow[t]{3}{*}{ Mitosis } & Low & $16(84.2)$ & $13(76.5)$ & $2(13.3)$ & $2(15.4)$ & $<.01$ \\
\hline & Intermediate & $1(5.3)$ & $4(23.5)$ & $3(20.0)$ & $3(23.1)$ & \\
\hline & High & $2(10.5)$ & 0 & $10(66.7)$ & $8(61.5)$ & \\
\hline \multirow[t]{2}{*}{ ER } & Negative & 0 & 0 & $5(33.3)$ & $4(30.8)$ & .04 \\
\hline & Positive & $19(100)$ & $17(100)$ & $10(66.7)$ & $9(69.2)$ & \\
\hline \multirow[t]{2}{*}{ PR } & Negative & $1(5.3)$ & $1(5.9)$ & $7(46.7)$ & $5(38.5)$ & .04 \\
\hline & Positive & $18(94.7)$ & $16(94.1)$ & $8(53.3)$ & $8(61.5)$ & \\
\hline \multirow[t]{2}{*}{ HER2 } & Negative & $18(94.7)$ & $16(94.1)$ & $9(60.0)$ & $8(61.5)$ & .01 \\
\hline & Positive & $1(5.3)$ & $1(5.9)$ & $6(40.0)$ & $5(38.5)$ & \\
\hline \multirow[t]{2}{*}{ Ki-67 } & Low & $11(57.9)$ & $7(41.2)$ & $1(6.7)$ & $1(7.7)$ & $<.01$ \\
\hline & High & $8(42.1)$ & $10(58.8)$ & $14(93.3)$ & $12(92.3)$ & \\
\hline \multirow[t]{4}{*}{ MS } & LA & $11(57.9)$ & $6(35.3)$ & $1(6.7)$ & $1(7.7)$ & $<.01$ \\
\hline & LB & $8(42.1)$ & $11(64.7)$ & $9(60.0)$ & $8(61.5)$ & \\
\hline & HER2 & 0 & 0 & $2(13.3)$ & $2(15.4)$ & \\
\hline & Basal-like & 0 & 0 & $3(20.0)$ & $2(15.4)$ & \\
\hline \multirow[t]{3}{*}{ T-stage } & 1 & $11(57.9)$ & $6(35.3)$ & $6(40.0)$ & $4(30.8)$ & .62 \\
\hline & 2 & $7(36.8)$ & $7(41.2)$ & $6(40.0)$ & 7 (53.8) & \\
\hline & 3 & $1(5.3)$ & $4(23.5)$ & $3(20.0)$ & $2(15.4)$ & \\
\hline \multirow[t]{2}{*}{ LNM } & Absence & 17 (89.5) & $13(76.5)$ & $3(20.0)$ & $4(30.8)$ & $<.01$ \\
\hline & Presence & $2(10.5)$ & $4(23.5)$ & $12(80.0)$ & $9(69.2)$ & \\
\hline
\end{tabular}

Values are presented as number (\%).

MUC, mucinous carcinoma without micropapillary feature; MUMPC, mucinous carcinoma with micropapillary feature; IMPC, invasive micropapillary carcinoma; IDMPC, mixed invasive ductal and micropapillary carcinoma; NG, nuclear grade; ER, estrogen receptor; PR, progesterone receptor; HER2, human epidermal growth factor receptor 2; MS, molecular subtype; LA, luminal A; LB, luminal B; LNM, lymph node metastasis.

${ }^{\mathrm{a} C h i-s q u a r e ~ t e s t ; ~}{ }^{\mathrm{b}} \mathrm{ANOVA}$ test. 
Table 2. Clinicopathological parameters related to death

\begin{tabular}{|c|c|c|c|c|}
\hline Parameter & & Alive & Dead & $p$-value \\
\hline Age, mean (yr) & & $51.6 \pm 12.3$ & $53.0 \pm 16.0$ & $.75^{b}$ \\
\hline \multirow[t]{4}{*}{ Category } & MUC & $19(100)$ & 0 & .20 \\
\hline & MUMPC & $14(82.4)$ & $3(17.6)$ & \\
\hline & IMPC & $12(80.0)$ & $3(20.0)$ & \\
\hline & IDMPC & $10(76.9)$ & $3(23.1)$ & \\
\hline \multirow[t]{2}{*}{ MUC vs MUMPC } & MUC & $19(100)$ & 0 & .06 \\
\hline & MUMPC & $14(82.4)$ & $3(17.6)$ & \\
\hline \multirow[t]{3}{*}{$N G$} & Low & $7(100)$ & 0 & .34 \\
\hline & Intermediate & $29(87.9)$ & $4(12.1)$ & \\
\hline & High & $19(79.2)$ & $5(20.8)$ & \\
\hline \multirow[t]{3}{*}{ Mitosis } & Low & 31 (93.9) & $2(6.1)$ & .14 \\
\hline & Intermediate & $8(72.7)$ & $3(27.3)$ & \\
\hline & High & $16(80.0)$ & $4(20.0)$ & \\
\hline \multirow[t]{2}{*}{ ER } & Negative & $6(66.7)$ & $3(33.3)$ & .07 \\
\hline & Positive & 49 (89.1) & $6(10.9)$ & \\
\hline \multirow[t]{2}{*}{ PR } & Negative & $9(64.3)$ & $5(35.7)$ & .01 \\
\hline & Positive & $46(92.0)$ & $4(8.0)$ & \\
\hline \multirow[t]{2}{*}{ HER2 } & Negative & 47 (92.2) & $4(7.8)$ & .01 \\
\hline & Positive & $8(61.5)$ & $5(38.5)$ & \\
\hline \multirow[t]{2}{*}{ Ki-67 } & Low & $19(95.0)$ & $1(5.0)$ & .16 \\
\hline & High & $36(81.8)$ & $8(18.2)$ & \\
\hline \multirow[t]{4}{*}{ MS } & LA & $20(82.9)$ & 0 & .05 \\
\hline & LB & $29(82.9)$ & $6(17.1)$ & \\
\hline & HER2 & $2(50.0)$ & $2(50.0)$ & \\
\hline & Basal-like & $4(80.0)$ & $1(20.0)$ & \\
\hline \multirow[t]{3}{*}{ T-stage } & 1 & 27 (100) & 0 & $<.01$ \\
\hline & 2 & $24(88.9)$ & $3(11.1)$ & \\
\hline & 3 & $4(40.0)$ & $6(60.0)$ & \\
\hline \multirow[t]{2}{*}{ LNM } & Absence & $36(97.3)$ & $1(2.7)$ & $<.01$ \\
\hline & Presence & $19(70.4)$ & $8(29.6)$ & \\
\hline
\end{tabular}

MUC, mucinous carcinoma without micropapillary feature; MUMPC, mucinous carcinoma with micropapillary feature; IMPC, invasive micropapillary carcinoma; IDMPC, mixed invasive ductal and micropapillary carcinoma; NG, nuclear grade; ER, estrogen receptor; PR, progesterone receptor; HER2, human epidermal growth factor receptor 2; MS, molecular subtype; LA, luminal A; LB, luminal B; LNM, lymph node metastasis. ${ }^{a}$ Chi-square test; ${ }^{b t}$ test (2-tailed).

type. ${ }^{2}$ In this study, pure mucinous carcinoma showed a low rate of nodal involvement $(16.7 \%)$ and a high rate of OS (91.7\%). This indolent behavior is associated with a relatively low level of genomic instability, low proliferative activity, positivity for hormone receptors, and low HER2 amplification. ${ }^{2,24,25}$ In the present study, pure mucinous carcinoma showed a low nuclear grade, diffuse and strong expression of hormone receptors, and very low expression of HER2.

A micropapillary architecture in breast carcinoma has been reported to result in a poor prognosis. ${ }^{17,18,26,27}$ In one study, $86 \%$ of mucinous carcinomas had a micropapillary pattern. ${ }^{28}$ The micropapillary architecture can be ignored in tumors with a large quantity of extracellular mucin as detected by low-power microscopy. ${ }^{16} \mathrm{~A}$ micropapillary pattern has been identified in pure mucinous carcinoma, although its prognostic significance is unclear. In this study, 17 of 36 cases of mucinous carcinoma (47.2\%) had micropapillary features. As reported in a previous study, these micropapillary subtypes of mucinous carcinoma impact patient survival via their propensity for nodal metastases, depending on the amount of mucin within the tumor. ${ }^{16,28}$ The study also demonstrated that even tumors classified as MUMPC can lead to IMPC-type metastasis. Prior studies have reported that mucinous carcinomas result in nonmucinous or ductal metastasis. ${ }^{29}$ However, the results are conflicting. In the current study, four of 17 MUMPC cases (23.5\%) had lymph node metastases of the ductal, micropapillary, or mucinous type. MUC and MUMPC were not significantly influenced by the presence of micropapillary features. It has been demonstrated in both types that abundant extracellular mucin contributes to the slower spread of pure mucinous carcinoma by functioning as a physical barrier between the neoplastic cells and surrounding stroma. ${ }^{30,31}$ This suggests that abundant mucin is a more important prognostic factor than is the presence of micropapillary features. The micropapillary pattern in mucinous carcinoma indicates a possible histogenetic association with IMPC. Indeed, the ability of IMPC to undergo at least partial mucinous differentiation has been reported. ${ }^{8,9,14}$

We separately reviewed 45 cases of breast carcinoma with micropapillary features, of which 17 cases were MUMPC, 15 IMPC, and 13 IDMPC, and found that nuclear grade; mitotic rate; ER, PR, HER2, and Ki-67 expression; and lymph node status differed significantly among the subtypes $(\mathrm{p}<.05)$. MUMPC and IMPC have been reported to have similar high nuclear grades, with 70\%-80\% having florid mitotic activity. ${ }^{10,11,18,32-34}$ This suggests that MUMPC and IMPC are components of the same spectrum; they show similar nuclear grades and vary only in their mucin content. In contrast, compared with patients with IMPC, those with MUMPC have a better prognosis irrespective of tumor stage. ${ }^{16}$ In the present study, MUMPC cases had lower and intermediate nuclear grades and a lower mitotic rate than those of IMPC cases. These results indicate that MUMPC exhibits a level of aggressiveness intermediate to those of pure mucinous carcinoma and IMPC.

Previous studies have reported high HER2 expression and low hormone receptor expression in IMPC of the breast. t, $15,17,27,32^{2}$ IMPC has a high propensity for lymph node metastasis and more frequent involvement of the lymph nodes compared with invasive ductal carcinoma. ${ }^{35}$ In this study, four cases of MUMPC (23.5\%) had lymph node metastasis; however, synchronous lymph node metastasis was detected in 12 cases of IMPC (80.0\%) and nine 
Table 3. Univariate and multivariate analyses of the associations between clinicopathological parameters and overall survival using logistic regression model

\begin{tabular}{|c|c|c|c|c|c|}
\hline & & Univariate & \multirow{2}{*}{ p-value } & Multivariate & \multirow{2}{*}{$p$-value } \\
\hline & & $\mathrm{RR}(95 \% \mathrm{Cl})$ & & $\mathrm{RR}(95 \% \mathrm{Cl})$ & \\
\hline \multirow[t]{3}{*}{ Category } & MUMPC & 1.0 & Reference & - & - \\
\hline & IMPC vs MUMPC & $1.2(0.2-6.9)$ & .87 & - & - \\
\hline & IDMPC vs MUMPC & $1.4(0.2-8.4)$ & .71 & - & - \\
\hline$N G$ & & $2.5(0.7-8.7)$ & .15 & - & - \\
\hline Mitosis & & $1.9(0.8-4.1)$ & .13 & - & - \\
\hline ER & & $0.3(0.1-1.2)$ & .09 & - & - \\
\hline PR & & $0.2(0.1-0.7)$ & .02 & $0.7(0.1-6.6)$ & .78 \\
\hline HER2 & & $7.3(1.6-33.0)$ & .01 & $1.1(0.1-11)$ & .92 \\
\hline Ki-67 & & $4.2(0.5-36.0)$ & .19 & - & - \\
\hline \multirow[t]{4}{*}{ MS } & LA & 1.0 & Reference & - & - \\
\hline & LB vs LA & 0.0 & .10 & - & - \\
\hline & HER2 vs LA & $4.8(0.6-41.0)$ & .15 & - & - \\
\hline & Basal-like vs LA & $1.2(0.1-13.0)$ & .88 & - & - \\
\hline T-stage & & $15(3.2-72.0)$ & .001 & $14(2.1-88)$ & .006 \\
\hline LNM & & $15(1.8-130.0)$ & .01 & $9(0.6-149)$ & .11 \\
\hline
\end{tabular}

$\mathrm{RR}$, relative risk; $95 \% \mathrm{Cl}, 95 \%$ confidence interval; MUMPC, mucinous carcinoma with micropapillary feature; IMPC, invasive micropapillary carcinoma; IDMPC, mixed invasive ductal and micropapillary carcinoma; NG, nuclear grade; ER, estrogen receptor; PR, progesterone receptor; HER2, human epidermal growth factor receptor 2; MS, molecular subtype; LA, luminal A; LB, luminal B; LNM, lymph node metastasis.

cases of IDMPC (69.2\%). In IMPC, histologic grade, lymphatic vessel density, and lymphocyte infiltration might influence lymph node metastasis. ${ }^{9,16,33,35,36}$ In the present study, MUMPC tumors showed high rates of hormone receptor expression: ER and PR were expressed in $17(100 \%)$ and $16(94.1 \%)$ of 17 cases, respectively; one case (5.9\%) was positive for HER2 amplification. A high rate of hormone receptor expression and a low rate of HER2 suggested good prognosis. Hsu and Shaw ${ }^{37}$ reported no HER2 amplification in mucinous breast cancer, suggesting that HER2 is rarely involved in its tumorigenesis; low HER2 amplification might also contribute to a better prognosis of this cancer.

Most cases of IMPC are associated with nodal metastases and a poor prognosis. ${ }^{12,13,34,38,39}$ There were typically multiple metastases, with $51 \%$ of cases having three or more positive lymph nodes. ${ }^{13,35}$ The average number of metastatic lymph nodes was shown to be $10.7 .^{38}$ In addition, lymphatic and vascular invasion has been reported in 33\%-67\% of cases. ${ }^{5,8,12}$ In this study, 12 of 15 IMPC cases $(80.0 \%)$ exhibited synchronous axillary lymph node metastasis. Of these 12 cases, multiple lymph node metastases $(>4)$ were noted in five cases (41.7\%). The mechanism underlying the high incidence of lymph node metastasis in IMPC is unclear, and the intrinsic subtype is considered an important prognostic factor. ${ }^{40,41}$

The prognosis of luminal A type breast cancer is markedly superior to that of HER2-positive and triple-negative breast cancers. A previous study reported that MUMPC revealed more prevalent luminal B or HER2 subtypes than MUC. ${ }^{42}$ However, we could not identify the clinical significance of breast carcinoma molecular subtype since there was no HER2 positivity in either MUC or MUMPC cases in this study.

In conclusion, our findings suggest heterogeneous biological behavior among tumors with a micropapillary architecture. Morphologically, MUMPC shares features with both mucinous carcinoma and IMPC, which could result in its intermediate clinical behavior. Although IMPC and IDMPC are aggressive tumors, the presence of micropapillary features in MUMPC was not associated with poorer prognosis. It can be postulated that MUMPC has a more favorable prognosis than that of IMPC, and that the micropapillary pattern in mucinous carcinoma does not contribute to its aggressive behavior. However, further analyses involving a larger cohort are required to clarify the pathogenetic relationships among these tumor types. Furthermore, the classical tumor stage was the strongest predictor of prognosis.

\section{Conflicts of Interest}

No potential conflict of interest relevant to this article was reported.

\section{Acknowledgments}

This work was supported by the 2012 Inje University research grant for Kyeongmee Park. 


\section{REFERENCES}

1. Barkley CR, Ligibel JA, Wong JS, Lipsitz S, Smith BL, Golshan M. Mucinous breast carcinoma: a large contemporary series. Am J Surg 2008; 196: 549-51.

2. Di Saverio S, Gutierrez J, Avisar E. A retrospective review with long term follow up of 11,400 cases of pure mucinous breast carcinoma. Breast Cancer Res Treat 2008; 111: 541-7.

3. Diab SG, Clark GM, Osborne CK, Libby A, Allred DC, Elledge RM. Tumor characteristics and clinical outcome of tubular and mucinous breast carcinomas. J Clin Oncol 1999; 17: 1442-8.

4. Louwman MW, Vriezen M, van Beek MW, et al. Uncommon breast tumors in perspective: incidence, treatment and survival in the Netherlands. Int J Cancer 2007; 121: 127-35.

5. Siriaunkgul S, Tavassoli FA. Invasive micropapillary carcinoma of the breast. Mod Pathol 1993; 6: 660-2.

6. Pettinato G, Manivel CJ, Panico L, Sparano L, Petrella G. Invasive micropapillary carcinoma of the breast: clinicopathologic study of 62 cases of a poorly recognized variant with highly aggressive behavior. Am J Clin Pathol 2004; 121: 857-66.

7. Tavassoli FA, Devilee P. World Health Organization classification of tumours: pathology and genetics of tumours of the breast and female genital organs. Lyon: IARC Press, 2003; 35-6.

8. Walsh MM, Bleiweiss IJ. Invasive micropapillary carcinoma of the breast: eighty cases of an underrecognized entity. Hum Pathol 2001; 32: 583-9.

9. Zekioglu O, Erhan Y, Ciris M, Bayramoglu H, Ozdemir N. Invasive micropapillary carcinoma of the breast: high incidence of lymph node metastasis with extranodal extension and its immunohistochemical profile compared with invasive ductal carcinoma. Histopathology 2004; 44: 18-23.

10. Paterakos M, Watkin WG, Edgerton SM, Moore DH 2nd, Thor AD. Invasive micropapillary carcinoma of the breast: a prognostic study. Hum Pathol 1999; 30: 1459-63.

11. Middleton LP, Tressera F, Sobel ME, et al. Infiltrating micropapillary carcinoma of the breast. Mod Pathol 1999; 12: 499-504.

12. Luna-Moré S, Gonzalez B, Acedo C, Rodrigo I, Luna C. Invasive micropapillary carcinoma of the breast: a new special type of invasive mammary carcinoma. Pathol Res Pract 1994; 190: 668-74.

13. Nassar H, Wallis T, Andea A, Dey J, Adsay V, Visscher D. Clinicopathologic analysis of invasive micropapillary differentiation in breast carcinoma. Mod Pathol 2001; 14: 836-41.

14. Luna-Moré S, Casquero S, Pérez-Mellado A, Rius F, Weill B, Gornemann I. Importance of estrogen receptors for the behavior of invasive micropapillary carcinoma of the breast. Review of 68 cases with followup of 54. Pathol Res Pract 2000; 196: 35-9.
15. Barbashina V, Corben AD, Akram M, Vallejo C, Tan LK. Mucinous micropapillary carcinoma of the breast: an aggressive counterpart to conventional pure mucinous tumors. Hum Pathol 2013; 44: 1577-85.

16. Liu F, Yang M, Li Z, et al. Invasive micropapillary mucinous carcinoma of the breast is associated with poor prognosis. Breast Cancer Res Treat 2015; 151: 443-51.

17. Ng WK. Fine-needle aspiration cytology findings of an uncommon micropapillary variant of pure mucinous carcinoma of the breast: review of patients over an 8-year period. Cancer 2002; 96: 280-8.

18. Madur B, Shet T, Chinoy R. Cytologic findings in infiltrating micropapillary carcinoma and mucinous carcinomas with micropapillary pattern. Acta Cytol 2007; 51: 25-32.

19. Yang YL, Liu BB, Zhang X, Fu L. Invasive micropapillary carcinoma of the breast: an update. Arch Pathol Lab Med 2016; 140: 799-805.

20. Edge SB, Byrd DR, Compton CC, Fritz AG, Greene FL, Trotti A. AJCC cancer staging manual. 7th ed. New York: Springer, 2009; 589628.

21. Hammond ME, Hayes DF, Dowsett M, et al. American Society of Clinical Oncology/College of American Pathologists guideline recommendations for immunohistochemical testing of estrogen and progesterone receptors in breast cancer. Arch Pathol Lab Med 2010; 134: 907-22.

22. Wolff AC, Hammond ME, Hicks DG, et al. Recommendations for human epidermal growth factor receptor 2 testing in breast cancer: American Society of Clinical Oncology/College of American Pathologists clinical practice guideline update. Arch Pathol Lab Med 2014; 138: 241-56.

23. Goldhirsch A, Wood WC, Coates AS, et al. Strategies for subtypes: dealing with the diversity of breast cancer: highlights of the St. Gallen International Expert Consensus on the Primary Therapy of Early Breast Cancer 2011. Ann Oncol 2011; 22: 1736-47.

24. Fujii H, Anbazhagan R, Bornman DM, Garrett ES, Perlman E, Gabrielson E. Mucinous cancers have fewer genomic alterations than more common classes of breast cancer. Breast Cancer Res Treat 2002; 76: 255-60.

25. Lacroix-Triki M, Suarez PH, MacKay A, et al. Mucinous carcinoma of the breast is genomically distinct from invasive ductal carcinomas of no special type. J Pathol 2010; 222: 282-98.

26. Bal A, Joshi K, Sharma SC, Das A, Verma A, Wig JD. Prognostic significance of micropapillary pattern in pure mucinous carcinoma of the breast. Int J Surg Pathol 2008; 16: 251-6.

27. Ranade A, Batra R, Sandhu G, Chitale RA, Balderacchi J. Clinicopathological evaluation of 100 cases of mucinous carcinoma of breast with emphasis on axillary staging and special reference to a micropapillary pattern. J Clin Pathol 2010; 63: 1043-7.

28. Shet $T$, Chinoy R. Presence of a micropapillary pattern in mucinous 
carcinomas of the breast and its impact on the clinical behavior. Breast J 2008; 14: 412-20.

29. Rasmussen BB, Rose C, Christensen IB. Prognostic factors in primary mucinous breast carcinoma. Am J Clin Pathol 1987; 87: 155-60.

30. Akiyoshi T, Nagaie T, Tokunaga M, et al. Invasive micropapillary carcinoma of the breast with minimal regional lymph node metastasis regardless of the huge size: report of a case. Breast Cancer 2003; 10: 356-60.

31. Anan K, Mitsuyama S, Tamae K, et al. Pathological features of mucinous carcinoma of the breast are favourable for breast-conserving therapy. Eur J Surg Oncol 2001; 27: 459-63.

32. Kuroda H, Sakamoto G, Ohnisi K, Itoyama S. Clinical and pathologic features of invasive micropapillary carcinoma. Breast Cancer 2004; 11: 169-74.

33. Guo X, Chen L, Lang R, Fan Y, Zhang X, Fu L. Invasive micropapillary carcinoma of the breast: association of pathologic features with lymph node metastasis. Am J Clin Pathol 2006; 126: 740-6.

34. Tresserra F, Grases PJ, Fábregas R, Férnandez-Cid A, Dexeus S. Invasive micropapillary carcinoma: distinct features of a poorly recognized variant of breast carcinoma. Eur J Gynaecol Oncol 1999; 20: 205-8.

35. Chen AC, Paulino AC, Schwartz MR, et al. Prognostic markers for invasive micropapillary carcinoma of the breast: a population-based analysis. Clin Breast Cancer 2013; 13: 133-9.

36. De la Cruz C, Moriya T, Endoh M, et al. Invasive micropapillary carcinoma of the breast: clinicopathological and immunohistochemical study. Pathol Int 2004; 54: 90-6.

37. Hsu YH, Shaw CK. Expression of p53, DCC, and HER-2/neu in mucinous carcinoma of the breast. Kaohsiung J Med Sci 2005; 21: 197-202.

38. Tsumagari K, Sakamoto G, Akiyama F, Kasumi F. The clinicopathological study of invasive micropapillary carcinoma of the breast. Jpn J Breast Cancer 2001; 16: 341-8.

39. Ide Y, Horii R, Osako T, et al. Clinicopathological significance of invasive micropapillary carcinoma component in invasive breast carcinoma. Pathol Int 2011; 61: 731-6.

40. Perou CM, Sorlie T, Eisen MB, et al. Molecular portraits of human breast tumours. Nature 2000; 406: 747-52.

41. Sorlie T, Perou CM, Tibshirani R, et al. Gene expression patterns of breast carcinomas distinguish tumor subclasses with clinical implications. Proc Natl Acad Sci US A 2001; 98: 10869-74.

42. Min SY, Jung EJ, Seol H, Park IA. Cancer subtypes of breast carcinoma with micropapillary and mucinous component based on immunohistochemical profile. Korean J Pathol 2011; 45: 125-31. 